\title{
Automatic Target Recognition (ATR) System using Recurrent Neural Network (RNN) for Pulse Radar
}

\author{
Kaustubh Bhattacharyya \\ Department of Electronics and Communication \\ Technology, Gauhati University, Guwahati- \\ 781014, Assam, India
}

\author{
Kandarpa Kumar Sarma \\ Department of Electronics and Communication \\ Technology, Gauhati University, Guwahati- \\ 781014, Assam, India
}

\begin{abstract}
The most fundamental problem in radar is the detection of an object or a physical phenomenon. This requires proper discrimination between signal and noise content at the receiver even after the echo containing target information is surrounded by clutter. Traditionally, a series of signal processing operations are carried out to perform this discrimination with varying levels of success. These series of signal processing operations can be supplemented by an Artificial Neural Network (ANN), which is a non-parametric prediction tool with the ability to retain the learning acquired from the surroundings. The Recurrent Neural Network (RNN) is a dynamic ANN which can track time variations in the input patterns. The RNN captures time-varying contextual information and use this knowledge subsequently to make discrimination between adjacent patterns. As viewed from the time domain, the target waveform can also be regarded as a time sequence such that it can be classified using RNN which is suitable for time sequence processing. This work describes the processing steps of signals from pulse radars so that these can be used to train a RNN for use to discriminate between target and false echoes. The experimental results show that the proposed system works effectively while dealing with target echoes surrounded by thermal noise and ground clutter at varying distances [1].
\end{abstract}

\section{Keywords}

Radar, target, recognition, recurrent, neural, network, clutter, processing

\section{INTRODUCTION}

The basic idea behind radio detection and ranging (RADAR) is to send out a signal and listen to an "echo" of this signal bouncing off from a target. The object's distance can be determined by the delay between the time that the signal was sent out and the time that it is received. Doppler shift can be used to determine the velocity of an object. It is defined as the difference between the frequencies of transmitted signal and reflected signal from moving objects. For range processing, the return signal must first be filtered in order to reduce the effects of clutter and noise that get in to the signal due to buildings, mountains, machinery and other signals. Radars are used in a wide variety of applications including defense, air traffic control, meteorology, speed monitoring and surveillance and even mapping for which precise discrimination between target and cluster is essential.

Working of the radar involves interpretation of returned signal to determine the characteristic of targets. With the unavoidable presence of various types of interference, signal processing is naturally an important part of any radar system. Signals recovered by the radar are composite form of target echoes and interference. The basic role of signal processing is to enhance the target echoes and suppress all other disturbances and extract information about the target's behavior, including its position, velocity, and signature. Signal processing exploits differences between the components of the composite signal [1], [2].

Automatic Target Recognition (ATR) is one of the major areas of modern radar signal processing. In this area, numerous approaches have been investigated. In recent years, considerable attention has been paid to waveform recognition techniques because of the simplicity and suitability. However, many research efforts are directed towards the development of the target recognition technique using high resolution radar returns [3].

Artificial Neural Network (ANN)s are computational tools that learn from experience (training), generalize from previous examples to new ones, and abstract essential characteristics from input containing irrelevant data [4]. Furthermore, due to their nonlinear nature, they can perform functional approximation and signal filtering operations, which are beyond optimal linear techniques [4].

A Recurrent Neural Network (RNN) is a class of ANN where connections between units form a directed cycle. This creates an internal state of the network which allows it to exhibit dynamic temporal behavior. Unlike feedforward ANNs, RNNs can use their internal memory to process arbitrary sequences of inputs.

This work describes a sequence of steps for processing of signals from pulse radars. These sequences are used as input features to a RNN for four radar target identification test cases. The targets are placed at different distances and pulse radar signals are continuously received. These returns are used to train the RNN which provides target class discrimination decision. The data set consists of target returns obtained from different distances under varying background conditions. The RNN receives inputs for a range of distance and data variations and learn them during training. Similar set of samples are used for testing its ability of target recognition under simulated conditions. Experimental results prove its effectiveness as an ATR system for pulse radars. The experimental results further show that the proposed system works effectively while dealing with radar returns surrounded by thermal noise and ground clutter.

Some relevant literatures are [5]-[12]. Very few attempts have been reported regarding the use of ANN for ATR design using pulse radars, hence the present work is a novel attempt in this regard.

The rest of the paper is organized as follows: Section 2 describes briefly about the theoretical aspects of the system components while Section 3 furnishes detail about the proposed model followed by the experimental results in 
Section 4. Section 5 gives a conclusion of the work described as part of the proposed system.

\section{THEORETICAL BACKGROUND}

Here, we briefly describe some theoretical aspects of the system components related to the work.

\subsection{Pulse Radar}

Pulse radar transmits a sequence of short pulses of RF energy. By measuring the time for echoes of these pulses scattered off a target to return to the radar, the range to the target can be estimated by the pulse radar. Here we consider a pulse radar transmitting between $10 \mathrm{GHz}$ to $12 \mathrm{GHz}$ frequency.

\subsection{Clutter}

The term "clutter" refers to any unwanted radar echo. Such echoes surrounded the radar signal and make it difficult to detect the presence of a target of interest [13]. The clutter is a disruptive factor in radar detection.

\subsection{Automatic Target Recognition (ATR)}

Automatic target recognition (ATR), is the ability of an algorithm or device to recognize targets or objects based on data obtained from radar return signals. The application of ATR technology is a critical element of radar signal processing.

\subsection{Artificial Neural Network (ANN)}

An ANN is an interconnected group of artificial neurons that uses a mathematical or computational model for information processing based on a connectionist approach of computation. In most cases an ANN is an adaptive system that changes its structure based on external or internal information that flows through the network. ANNs can identify and learn correlated patterns between input data sets and corresponding target values.

\subsection{Multilayer Perceptron (MLP)}

An MLP consists of several layers of neurons. The expression for output in a MLP with one hidden layer is given as:

$$
\mathbf{O}_{\mathrm{X}}=\boldsymbol{\beta}_{\mathrm{i}} \mathbf{g}\left(\left([\mathbf{w}]_{\mathrm{i}} \cdot[\mathbf{x}]\right)+\mathbf{b}_{\mathrm{i}}\right)
$$

where $\boldsymbol{\beta}_{\mathbf{i}}$ is the weight value between the $\mathrm{i}^{\text {th }}$ hidden neuron, $[\mathrm{w}]$ is the vector of weights between the input and the hidden layer, $[\mathrm{x}]$ is the vector of inputs and $[\mathrm{b}]$ is the input bias of the hidden neuron layer. Such a set- up is depicted in Figure 1.

MLPs are commonly used to approximate complex nonlinear mappings.

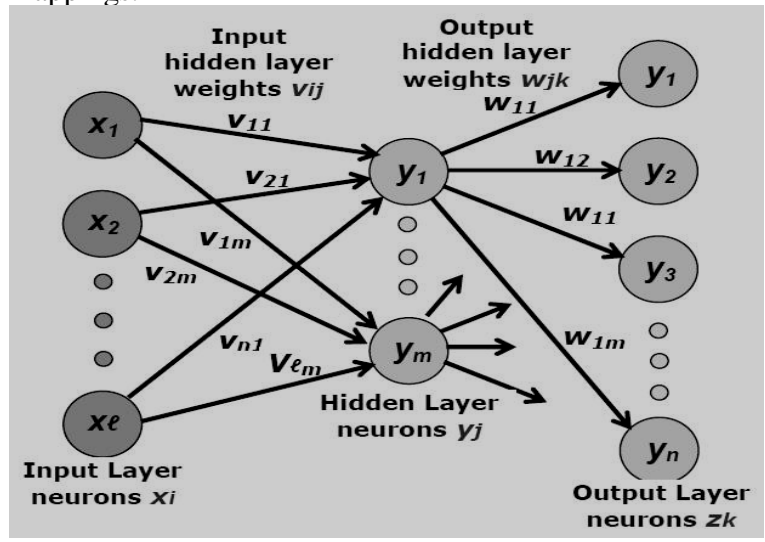

Figure 1: MLP with one input, one hidden and one output layer

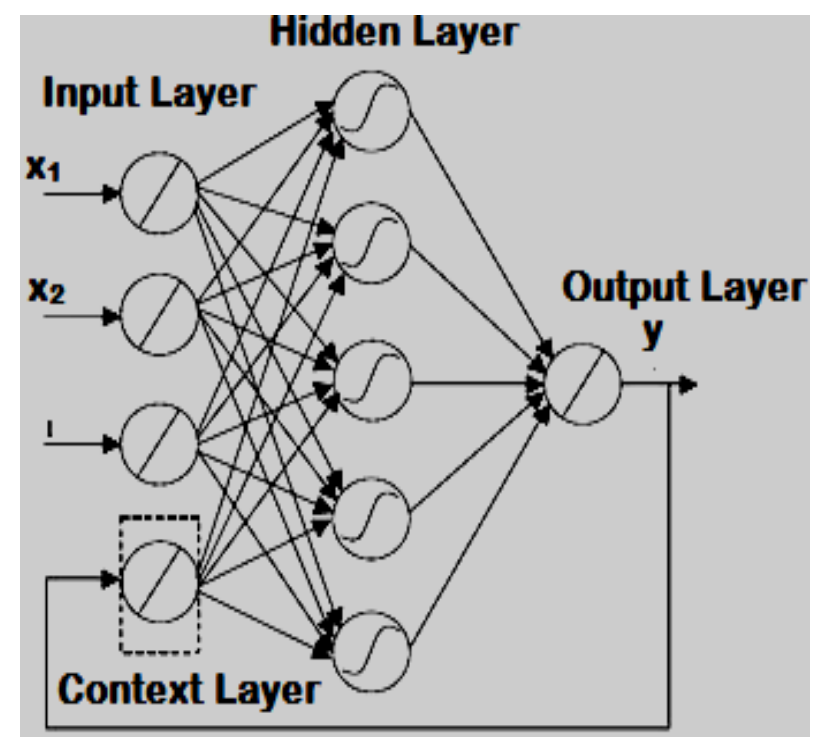

Figure 2: A simple RNN model

\subsection{Recurrent Neural Network (RNN)}

A RNN is a modification of feed forward architecture to allow for temporal classification, as shown in Figure 2. In this case, a "context" layer is added to the structure, which retains information between observations. At each time step, new inputs are fed into the RNN. The previous contents of the hidden layer are passed into the context layer. These then feed back into the hidden layer in the next time step. The context layer is formed by certain feedback mechanism which enables the RNN to track the variations in input patterns. The feedback mechanism further makes the RNN dynamic system suitable for ATR.

\section{PROPOSED RNN BASED ATR SYSTEM}

The system model of the proposed RNN based ATR is shown in Figure 3. 


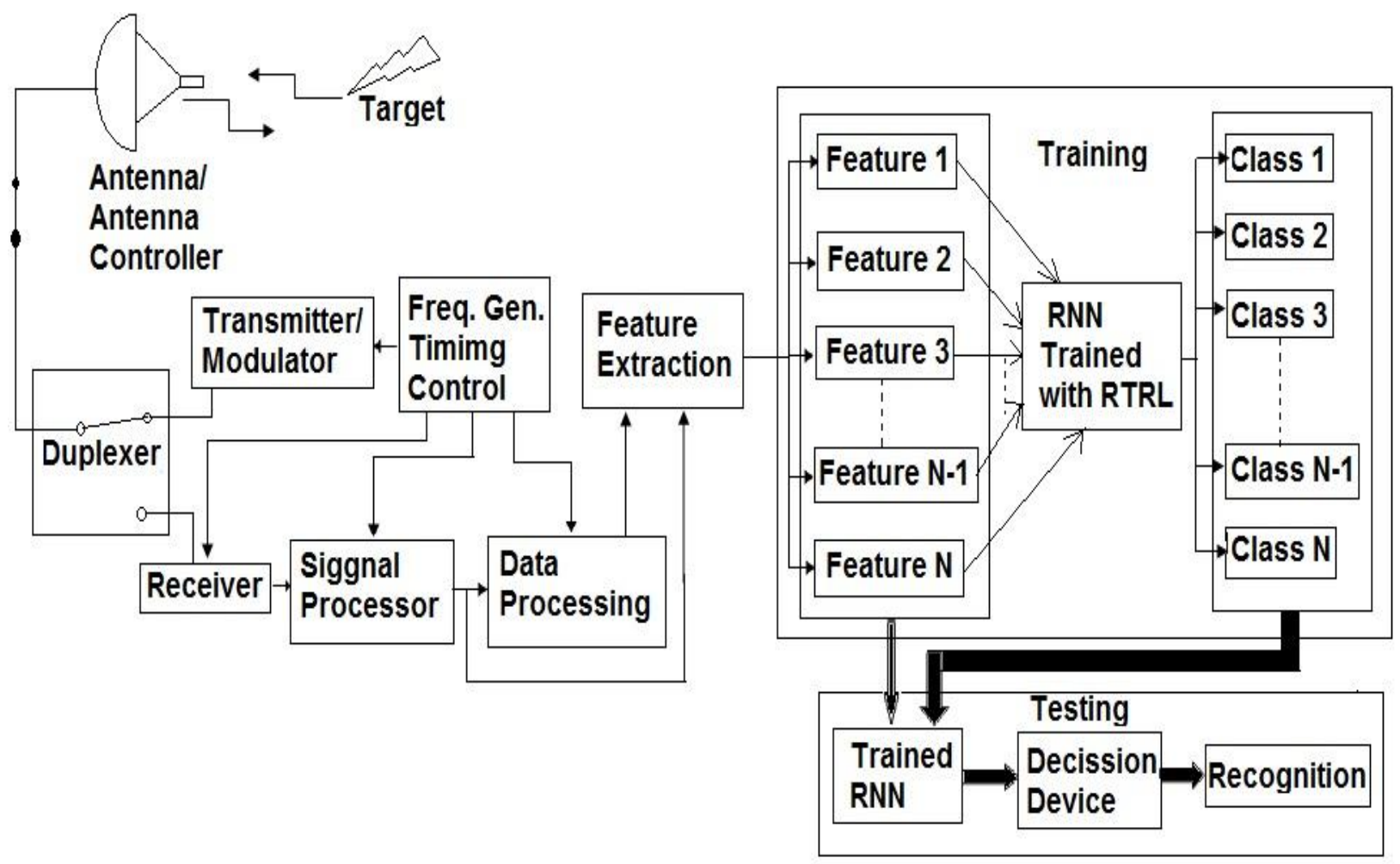

Figure 3: System model of the proposed RNN based AT R System

The work is constituted by an RNN which performs target recognition using returns received from a pulse radar. The training data set is prepared by certain efficient signal processing steps. The algorithm involves, first, the generation of a fast/slow-time matrix of raw baseband in-phase (I) and quadrature-phase $(\mathrm{Q})$ radar data that contains returns from exactly four targets (stationary) as well as interference (noise and clutter). A few radar signal processing steps allow us to prepare the appropriate data to train the RNN to detect each of the targets. These are described bellow in Section 3.1.

\subsection{Steps Involved In Signal Processing} Steps of the Proposed ATR System:

1. Signal Integration collects and accumulates composite signals within the same range bin for several hits. The components which are ordered from hit-to-hit produce a larger sum than those which are random.

2. Correlation is the process of measuring the similarity between two functions. Signal and interference are compared to a cost function designed to discriminate between a true and false target. The degree of match gives the likelihood that the composite signal contains a target echo. Pulse compression is the process of correlation in time domain which increases signal content in returned waveform.

3. Filtering and spectrum analysis is a frequency domain process, which accepts composite forms of target echoes and interference with a number of complex sinusoids, separating the signals in to their frequency components. The likelihood that a signal component represents the desired target depends on its spectrum.

In addition to those given above, two other processes are used to assist in performing the basic functions and suppressing processing artifacts.
4. Windowing: For processing, signals must be limited in time and the process should be finite. The result is processing errors that cause signals which occupy one output bin that spreads into other bins. This anomaly, called spectral leakage, degrades the output and can allow strong interfering signals to mask weaker target echoes [14].

5. Convolution in a domain (time, say) has the same effect as the multiplication in the other domain (frequency). In spectrum analysis, for example, the window is applied by multiplying in the time domain. It can, however, be applied, instead, in the frequency domain by using convolution.

\subsection{Interference}

The data contains two forms of interference:

- Thermal receiver noise, which is modeled as independent, but with unity variance, zero-mean white Gaussian noise processes in both the in-phase (I) and quadrature phase (Q) channels. It is completely uncorrelated from one pulse (slow-time sample) to the next and from one range bin (fast-time sample) to the next [15].

- Distributed ground clutter is highly correlated, and is usually removed by pre-filtering with a canceller. It is often considered independent from one range bin (fasttime) to the next, or nearly so, on the grounds that each range bin represents returns from physically distinct clutter. In this work, the ground clutter is created by forming a white random process having a log-normal amplitude distribution (typical of some types of real clutter) and uniform random phase. The desired correlation from one pulse to the next in a fixed range bin is created by forcing the spectrum of the samples from each range bin to have a narrow Gaussian shape. The clutter remains uncorrelated, however, from one range bin to the next [15]. 
For a single individual pulse, the signal to noise ratio (SNR), and/or signal to clutter ratio (SCR) of the echoes from some targets may be less than one. Therefore, the uncompressed pulse may be below the interference and can only be identified after some signal processing operations. On the other hand, clutter filtering, if used, will eliminate stationary targets. Such targets, if any, therefore, will not be detected unless we look for them before clutter filtering. Returns from stationary targets are large enough to stand out above the clutter, hence require efficient signal processing. Some of these issues are handled effectively by the ANN. Such performance becomes even more effective when a dynamic ANN like RNN is used. This aspect is covered in detail in section 3.3.

\subsection{Rnn Based Recognition}

Our problem is to predict the returns as well as the target structure using RNN classifier. To perform this task, we use RNN which have two important steps-training and testing.

Several RNN configurations are used to ascertain the best combination for the testing. RNNs use a form of BP called real-time recurrent learning (RTRL). For different training methods it shows different results. The number of training sessions and used data sets determines the outcome of the network. During training mean square error (MSE) convergence and prediction precision are used to ascertain the performance of the network. The trained RNNs are used for testing. Samples are used with variance up to 50 percent. Variance is essential to make the classifier robust. Prediction of radar target/returns primarily consists of two steps

- Derivation of pattern vector (from the radar data primarily for different ranges, frequency and RCS) through proper signal processing and

- Formation of decision logic for above parameters using RNN as single level classifier.

Here, we have taken four targets namely Target1, Target2, Target 3 and Target 4 as shown in Figure 4. These are, further, considered to be at different ranges. A pulse radar is simulated to transmit signals at these targets at different ranges. The return pulses are taken and used for training the RNNs for classification with different class-codes. The simulated conditions are similar to that shown in Figure 4. Radar Cross Section (RCS) values of the four targets are calculated for the considered conditions and used for RNN training configured to map the information to specific codes. The pattern association mapping maybe shown as in Figure 5.

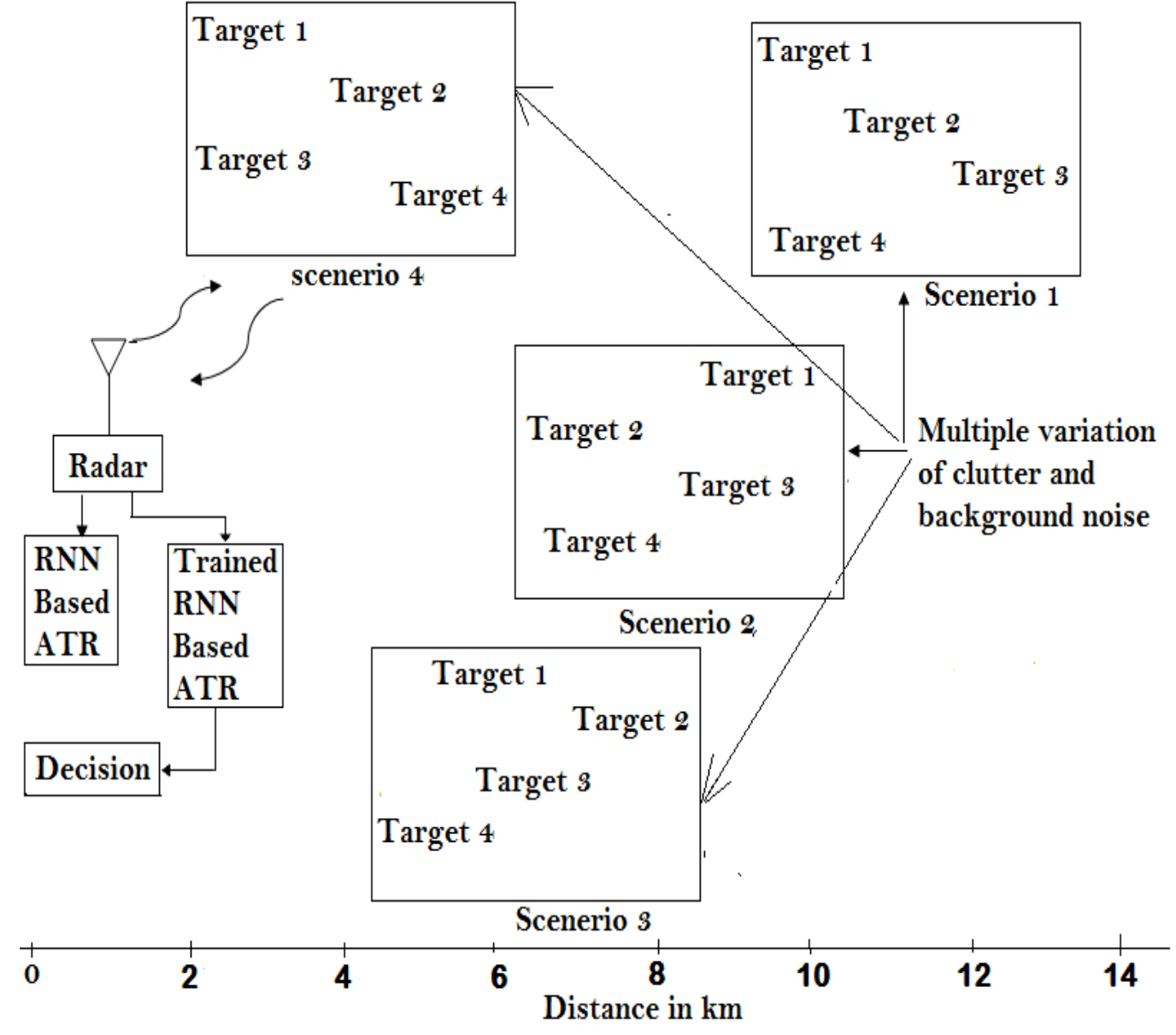

Figure 4: Considered Scenario 


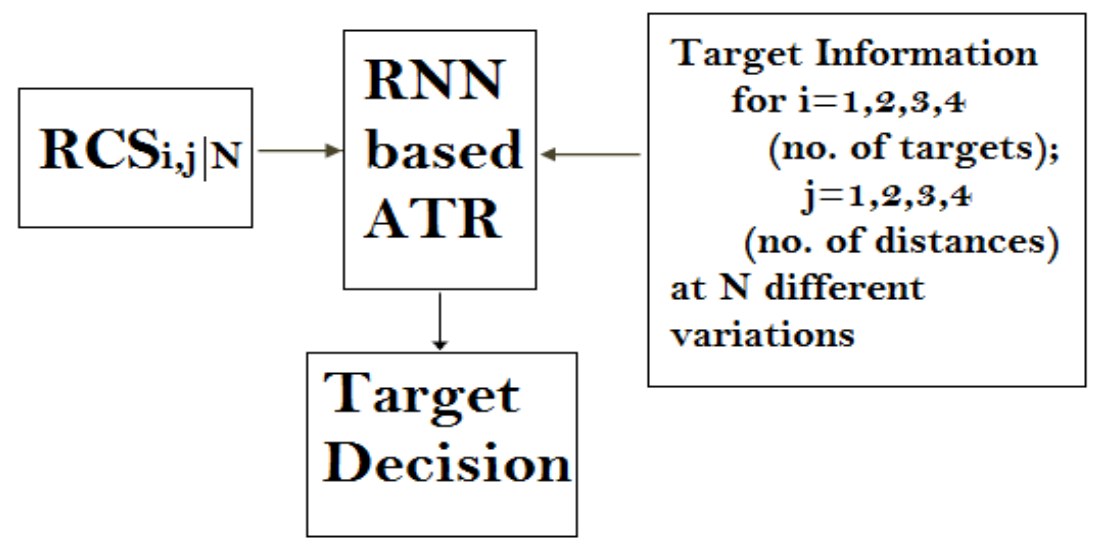

Figure 5: pattern association mapping

\section{EXPERIMENTAL RESULTS}

Table 1 gives the values of the range, SNR and relative RCS parameters used to create the data files. A more useful view is to plot the generated data is a range Doppler plot. This is obtained by transforming the fast time/slow time matrix in the slow time dimension to obtain a range-Doppler matrix.

Table 1 Range, velocity, and relative RCS parameters used to create the training data

\begin{tabular}{|l|l|l|l|}
\hline Target & Range & SNR & Real_RCS \\
\hline 1 & $2 \mathrm{~km}$ & $-3 \mathrm{~dB}$ & $-26.7 \mathrm{~dB}$ \\
\hline 2 & $3.8 \mathrm{~km}$ & $5 \mathrm{~dB}$ & $-7.55 \mathrm{~dB}$ \\
\hline 3 & $4.4 \mathrm{~km}$ & $10 \mathrm{~dB}$ & $0 \mathrm{~dB}$ \\
\hline 4 & $4.4 \mathrm{~km}$ & $7 \mathrm{~dB}$ & $-3 \mathrm{~dB}$ \\
\hline
\end{tabular}

We have not considered false alarms in this particular data set. To detect the range bins for targets, we have non-coherently integrated all of the Doppler bins to get a single column of total power vs. range. Then we implemented a logical search for local peaks above the constant false alarm rate (CFAR) threshold in range and recorded their range bins. The result of these operations is shown in Figure 6. At this point, our algorithm has generated the values of targets, which is given in Table 2. Now, the step involved in the work depicted by the second part of Figure 3 is carried out.

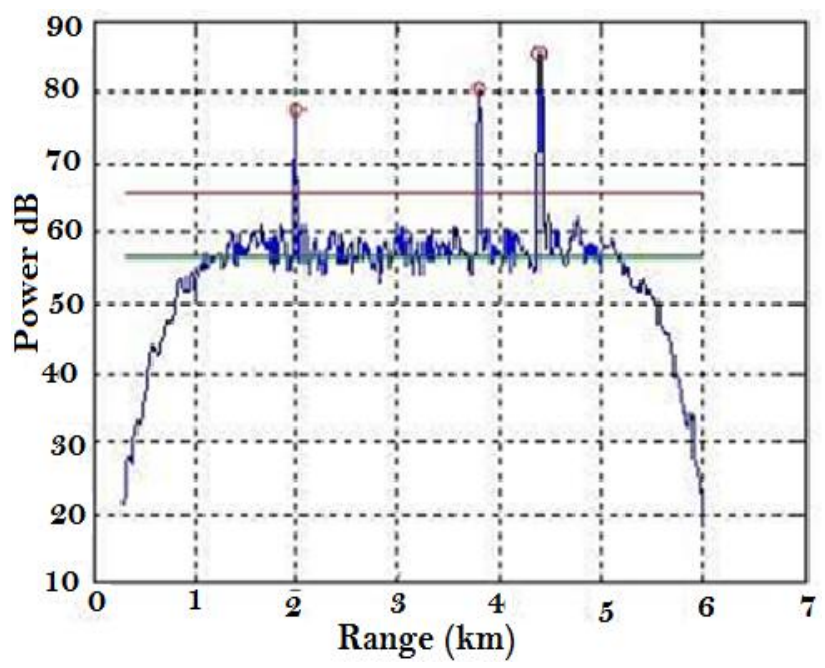

Figure 6: Detection of range peaks
Table 2: Calculated parameters for four targets

\begin{tabular}{|c|c|c|c|}
\hline Target & $\begin{array}{c}\text { Normalized } \\
\text { Power }\end{array}$ & $\begin{array}{c}\text { Range } \\
(\mathrm{km})\end{array}$ & DFT Index \\
\hline 1 & $0.4329 \times 10^{3}$ & 2 & 26.91 \\
\hline 2 & $0.6094 \times 10^{3}$ & 3.8 & 193.4 \\
\hline 3 & $1.00 \times 10^{3}$ & 4.4 & 51.8 \\
\hline 4 & $0.4471 \times 10^{3}$ & 4.4 & 168.2 \\
\hline
\end{tabular}

The high performance RTRL with variable learning rate is employed for training the RNN. The specifications of the ANN used for training phase are as tabulated in Table 3. The input layer consists of raw data, mixed up with thermal noise of unit power and mixed up with ground clutter and false echo.

We have carried out the training using tan sigmoidal for input, log sigmoidal for hidden and tan sigmoidal for output layer, activation functions in the network. The convergence of MSE for different number of iterations is shown in the following Figures 7 to 12 .

Table 3: Specifications of the ANN

\begin{tabular}{|l|l|}
\hline \multicolumn{2}{|c|}{ Type: RNN with RTRL } \\
\hline Number of Layers & \multicolumn{1}{|c|}{ Specifications } \\
\hline Lumber of Input Unit & $\begin{array}{l}\text { Data Matrix consisting of } \\
\text { vectors of four targets at } \\
\text { four different distances. }\end{array}$ \\
\hline Number of Output Unit & $\begin{array}{l}1 \text { binary encoded matrix } \\
\text { consisting of four target } \\
\text { vectors for four targets at } \\
\text { four different ranges. }\end{array}$ \\
\hline $\begin{array}{l}\text { Number of Neurons in the } \\
\text { Input Layer }\end{array}$ & 337 (size of the data matrix) \\
\hline $\begin{array}{l}\text { Number of Neurons in the } \\
\text { Hidden Layer }\end{array}$ & $337 * 1.5=506$ \\
\hline $\begin{array}{l}\text { Number of Neurons in the } \\
\text { Output Layer }\end{array}$ & 4 \\
\hline Number of Iterations & 20000 \\
\hline $\begin{array}{l}\text { Number of Validation } \\
\text { Checks }\end{array}$ & 6 \\
\hline Learning Rate & Adaptive \\
\hline Momentum & 0.9 \\
\hline Activation Functions & $\begin{array}{l}\text { Log-Sigmoid and Tan- } \\
\text { Sigmoid }\end{array}$ \\
\hline
\end{tabular}




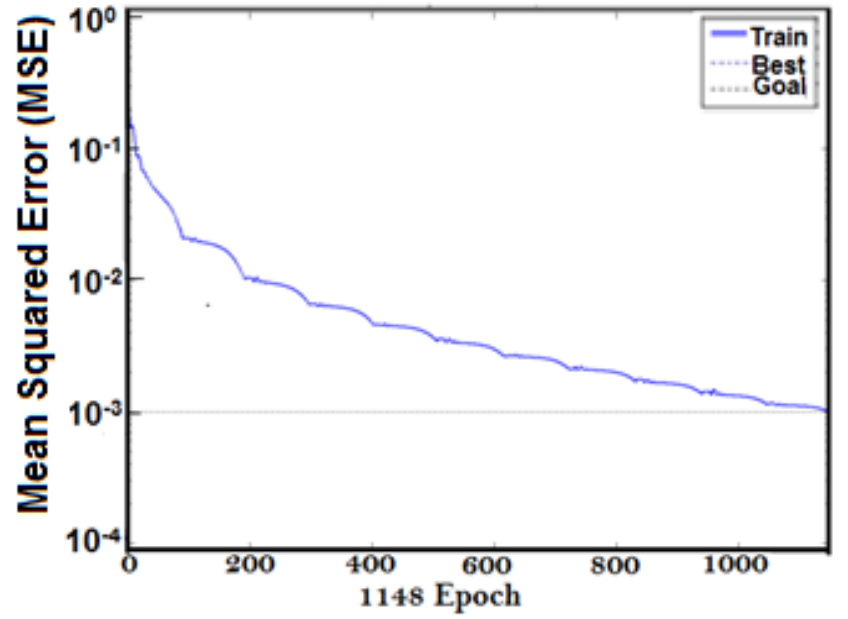

Figure 7: MSE curve for the RNN trained with raw data mixed with thermal noise and ground clutter

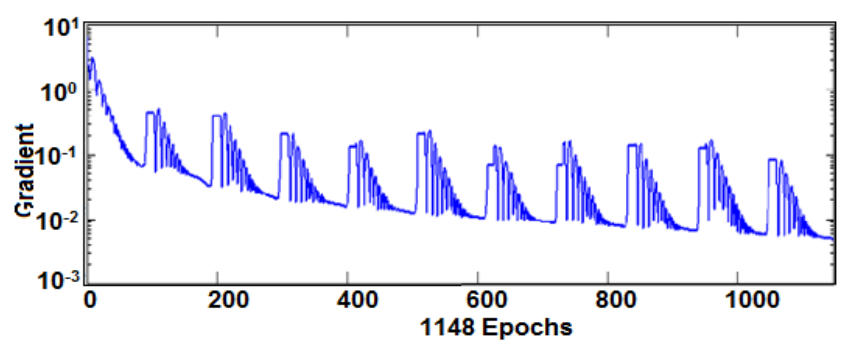

Figure 8: Gradient v/s iteration for the RNN trained with raw data mixed with thermal noise and ground clutter

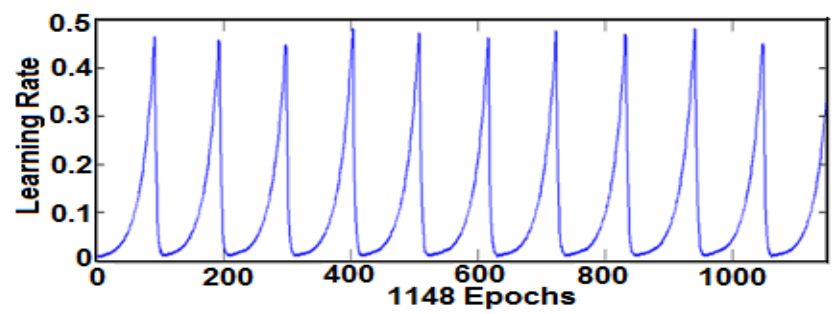

Figure 9: Learning rate v/s iteration for the RNN trained with raw data mixed with thermal noise and ground clutter

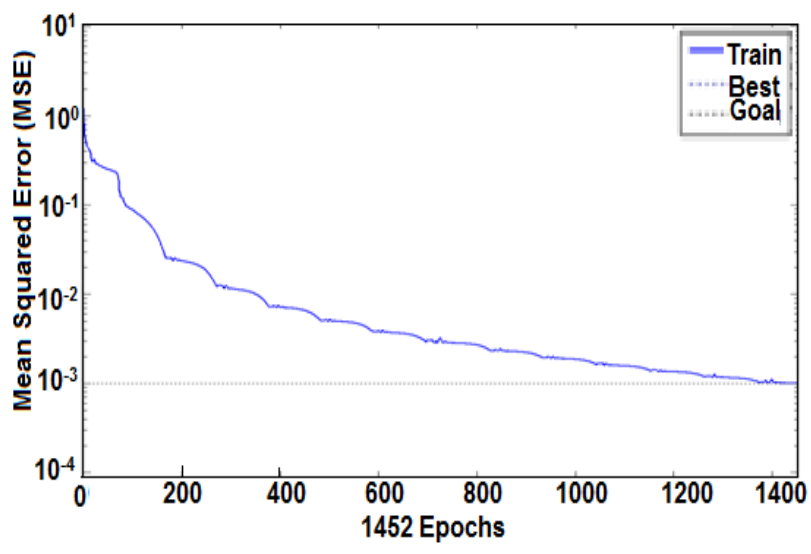

Figure 10: MSE curve for RNN trained raw data mixed with thermal noise and ground clutter along with separate ground clutter

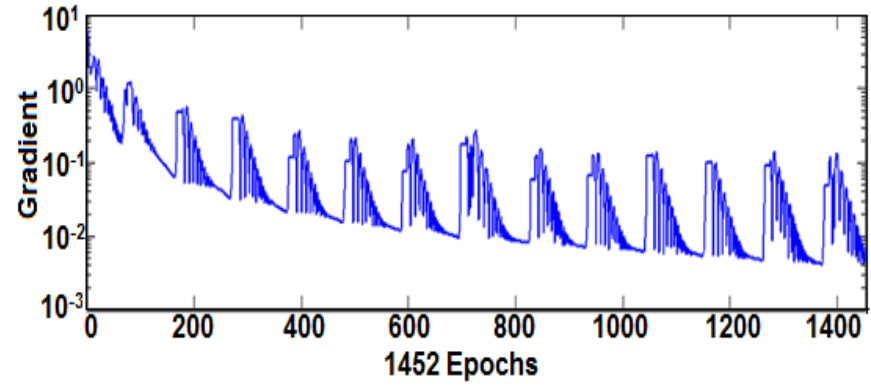

Figure 11: Gradient v/s iteration for RNN trained raw data mixed with thermal noise and ground clutter along with separate ground clutter

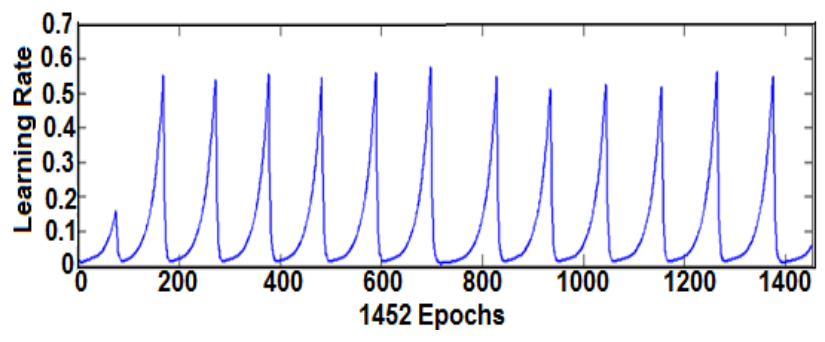

Figure 12: Learning rate v/s iteration for RNN trained raw data mixed with thermal noise and ground clutter along with separate ground clutter

The RTRL based training of the RNN generates the best classification performance. The number of training sessions has been restricted between 500 to 2000 sessions. This is because with training epochs below 500 the RNNs don't develop the ability to make discrimination between classes with success rates above $50 \%$. Again with training sessions over 2000, there is always a chance of the RNNs losing the ability to generalize classes.

The following two tables (Table 4 and Table 5) includes the performance parameters with variation in the number of pulses while training the RNN.

Table 4: Received signal without noise

\begin{tabular}{|l|l|l|}
\hline No. of pulses & CRR (\%) & FAR (\%) \\
\hline 5 & 68 & 32 \\
\hline 10 & 77 & 23 \\
\hline 20 & 82 & 18 \\
\hline
\end{tabular}

Table 5: Received signal with noise

\begin{tabular}{|l|l|l|}
\hline No. of pulses & CRR (\%) & FAR (\%) \\
\hline 5 & 57 & 43 \\
\hline 10 & 66 & 34 \\
\hline 20 & 73 & 27 \\
\hline
\end{tabular}

Here, we study the response in the performance of the system with changes in the number of the training targets. We notice an increase in the CRR of the system with the increase in the number of the training targets employed in training phase. The system provides $73 \%$ to $82 \%$ CRR with range and background variation with pulse radars. This performance can be further with better organization of the model mostly with stress on distributed and modular designs. 


\section{CONCLUSION}

The work offers an insight into the development of a RNN based system for prediction of target from radar return signal. The work improves the performance of ATR with signal processing. The system tackles well clutter mixed signals with range variations. The most striking feature of the work is the use of RNN which enables the system to capture time variation in the inputs which generates a CRR of above $82 \%$. It makes the system suitable for ATR applications using pulse radar returns. The difficulties associated with RNNs includes dimensionality and generalization problem. For learning many dimensional data such as radar return, either a huge number of samples is required, or the data must first be reduced by the extraction of a relatively small numbers of features for the RNN to learn. If the number of data samples available is too small, then the network will over fit the training data, resulting in poor generalization on test data. Hence, designing a RNN requires experimentation to determine the best architecture for a given problem and associated data set. The time required to train the system with the acquired data is found to be very high. This can be further reduced with high performance computational framework, although the system is required to be trained only once. The system in modular or distributed design shall provide improve results that the present ones.

\section{REFERENCES}

[1] M. I. Skolnik, "Opportunities in radar-2002," Electronic, Communication and Engineering Journal (ECEJ), IEE, PP. 263-272, December, 2002.

[2] A. Graps, "Radar development at Lincoln Laboratory: a fifty-year review, ” MIT-Lincoln Laboratory Journal, vol. 12, no. 2, PP. 139-444, 2000.

[3] F Qiang and Y Wenxian ATR National Lab. "Automatic target recognition based on incoherent radar returns" IEEE, PP. 123-128

[4] S. Haykin, "Neural Networks", Pearson Education, New Delhi, 2003.

[5] A Z Botros and A D Olver "Analysis of Target Response of FM-CW Radar," IEEE Transactions On Antennas And Propagation, Vol. AP-34, April, 1986.
[6] K Copsey and A Webb, "Bayesian Gamma Mixture Model Approach to Radar Target Recognition," IEEE Transactions on Aerospace and Electronic systems, Vol. 39, Oct. 2003.

[7] M. I. Kun-Mu Chen and B. Drachman, "Radar Target Discrimination by Convolution of Radar Return with Extinction-Pulses and Single-Mode Extraction Signals, , IEEE Transactions On Antennas And Propagation, Vol. AP-34, July, 1986.

[8] N. B. S. Chakrabarti and K. Theagharajan, "Robust Radar Target Classifier Using Artificial Neural Networks", IEEE Transactions On Neural Networks, Vol. 6, no. 3, May, 1995.

[9] E Avci, I Turkoolu and M Poyraz, "An Intelligent Target Recognition System Based On Periodogram For Pulsed Radar Systems" G.U. Journal of Science, Vol. 18, No. 2, Pp 259-272, 2005.

[10] S. Miyahara, "Automated Radar Target Recognition Based On Models Of Neural Nets," Dissertations available from ProQuest. Paper AAI8725190, January 1987.

[11] S. K Rogers, J. M Colombi, "Automatic target recognition using neural networks," Neural Networks, Vol 8, pp. 1153-1184, January 1995.

[12] L A Chan and N M Nasrabadi, "Automatic target recognition using vector quantization and neural networks, " Optical Engineering, Vol 38, no. 1, pp. 2147 2161,1999

[13] M. I. Skolnik, "Introduction to radar systems", 3rd ed., Mc Graw Hill, 2001

[14] F. E. Nathanson, "RADAR Design Principles" $2^{\text {nd }}$ Edition, McGraw Hill International Edition, Electrical Engineering Series, 1990

[15] S. Haykin, "Radar Signal Processing”, IEEE ASSP Magazine, April, 1985. 